\title{
Current view on biomedical applications of synthetic metal-polymer nanocomposites
}

\author{
Kaushik R, Sharma NK \\ Inderprastha Engineering College (IPEC), Ghaziabad, Uttar Pradesh, India
}

\begin{abstract}
Recent advances in the field of nanotechnology led several groups to recognize the promise of recruiting nanocomposities to the ongoing battle against pathogenic gram-positive and gramnegative bacteria and metal-polymer nanocomposites are a typical example. On the basis of recent literature, the nanocomposities are synthesized mainly by two techniques i.e. in-situ and ex-situ technique and these nanocomposities have great biomedical applications in terms of their antibacterial activity. A large battery of newly developed and discovered nanocomposities has been accumulating during the last decade.
\end{abstract}

Key words: Metal-polymer Nanocomposites; Synthesis; Characterization; Biomedical applications; Antibacterial activity

\section{INRODUCTION}

Modern technologies continuously need new materials with special combination of properties. Many efforts have been made in the last decades using novel nanotechnology and nanoscience knowledge in order to obtain nanocomposities with determined biomedical use [1], [2]. The Metal-polymer nanocomposites are a typical example of using nano-particles (nanostructured metal) as additives in polymer matrix. Recently, two techniques are being used to obtain metalpolymer nanocomposites. First, in-situ technique nanoparticles are generated inside polymer matrix by decomposition of metal precursors and first monomer is polymerized in solution containing metal ions chemical species and then metal nanoparticles are obtained reducing metal ions through chemical, thermal or photolysis methods [3]. Second technique is ex-situ technique in which nanoparticles are produced first by chemical methods then are distributed into a polymer solution or monomer solution to polymerize [4].

Development of new nanocomposites by using various materials is highly demanding area by advance techniques. These nanocomposites have tremendous applications in their respective fields. Out of traditional combination of constitutes, bulk nanocomposites materials are most rapidly moving field in material science since they can be designed from nontraditional combinations of the constituents. Apart from any discipline, chemistry has played a major role in developing new materials with novel and technologically important properties. The 
advantage of chemical synthesis is its versatility in designing and synthesizing novel nanocomposites that can be refined into the final product. The primary advantage that chemical processes offer over other methods is good chemical homogeneity, as chemical synthesis offers mixing at the molecular level. Molecular chemistry can be designed to prepare new materials by understanding how matter is assembled on an atomic and molecular level and the consequent effects on the desired material properties [5].

This rapidly expanding field "Nanotechnology" is generating many exciting new polymer-metal nanocomposites with novel properties. The later can be derived by combining properties from the parent constituents into a single material. There is also the possibility of new properties which are unknown in the parent constituent materials [6]. Nanocomposites combine favorable features of the constituents on the nanoscale to obtain new functionalities. The article will be concerned with the synthesis techniques (in-situ and ex-situ technique) of polymer-based nanocomposites consisting of metal nanoparticles in a polymer matrix and the resulting biomedical application especially antibacterial properties. Many researchers have contributed in development of various metal-polymer nanocomposites with antibacterial activity. Current examples are discussed regarding their synthesis and antibacterial activity, as biomedical application.

\section{SYNTHESIS OF METAL-POLYMER NANOCOMPOSITES}

There have been several attempts to achieve nanoparticle-polymer composites. According to current studies, researchers used two main techniques to prepare the polymer-metal composites. The first technique consists of the in-situ preparation of the nanoparticles in the polymer matrix. This is affected by the reduction of metal salts dissolved in the polymer matrix $[7,8]$. The second technique involves polymerizing the matrix around the nanoparticles and first nanoparticles are produced by chemical methods then are distributed into a polymer solution or monomer solution to polymerize. The technique is known as ex-situ technique [9, $10]$.

The development of uniform nanometer sized particles has been intensively pursued because of their technological and fundamental scientific importance. Nanoparticles present several different morphologies (flakes, spheres, dendritic shapes, etc.). They are generally designed and manufactured with physical properties tailored to meet the needs of the specific application they are going to be used for. There are numerous reported synthetic techniques for the production of nanoparticles. The challenge is to control the nanoparticle size, size distribution, morphology, crystallinity, shape and properties, to assemble the nanoparticles for a given purpose, and to make them from a variety of materials. Synthetic chemical methods for making metallic nanoparticles of controlled size and shape are continually being improved. Metals such as silver, gold, copper, cobalt, zinc and platinum have been made into nanospheres, nanorods, nanowires, nanocubes, and nanoprisms $[7,9$, $10,11]$.

Nanoparticles of different nature and size can be combined with polymers, give rise to a host of nanocomposites with interesting physical properties and important application potential. In 2001, Kumar et al., reported that composite materials containing amorphous 
$\mathrm{Cu}$ nanoparticles and nanocrystalline $\mathrm{Cu}_{2} \mathrm{O}$ embedded in polyaniline matrices were prepared by a sonochemical method. These composite materials were obtained from the sonication of copper (II) acetate when aniline or $1 \% \mathrm{v} / \mathrm{v}$ aniline-water was used as solvent. The physical and thermal properties of the as prepared composite materials were presented and mechanisms for the formation of these products were discussed. They are in opinion that this technique can also be applied to the preparation of different metals or metal oxides embedded in polyaniline matrices [12]. Gangopadhyay and De (2000) have been discussed in detail on the nanocomposites, throwing light on their synthesis techniques, properties and applications [13].

\section{CHARACTERIZATION OF METAL-POLYMER NANOCOMPOSITES}

Nanostructure of the nanocomposites usually consists of the matrix material containing the nanosized reinforcement components in the form of metal particles, whiskers, fibers, nanotubes, etc. Different investigators have employed various equipments and techniques for their identification and characterization of nanocomposites, including atomic force microscopy (AFM), scanning tunneling microscopy (STM), Fourier transformed infrared spectroscopy (FTIR), X ray photoelectron spectroscopy (XPS), nuclear magnetic resonance (NMR), differential scanning calorimetry (DSC), scanning and transmission electron microscopy (SEM/TEM), etc [14].

\section{BIOMEDICAL APPLICATION OF METAL- POLYMER NANOCOMPOSITES}

Metal-polymer nanocomposites are receiving great scientific interest for their effectiveness in catalysis and antimicrobial activity [15]. The antimicrobial potential of metal nanoparticles $(\mathrm{Ag}, \mathrm{Cu}$, etc.) allures the global scientific community to use this property for the welfare of human being. The metalpolymer nanocomposites have attracted the close scrutiny and considerable interest in both the academia and industry. This scrutiny results from the simple premise that using building blocks with dimensions in the nanosize range upon the addition of small amounts makes it possible to design and create new materials with improved physical properties and numerous potential applications such as enhancement of conductivity, toughness, optical activity, catalytic activity, chemical selectivity etc [16]. More recently, several nanomaterials including silver nanoparticles(nAg), photocatalytic $\mathrm{TiO} 2, \mathrm{ZnO}$, chitosan, aqueous fullerene nanoparticles (nC60), and carbon nanotubes (CNT), have shown strong antibacterial properties [3].

Biomedical application of the nanocomposities against pathogenic bacteria is a crucial step in the ongoing battle against these microbes because the increased development of new antibacterial agents. Here we will focus on two specific examples of biomedical approaches, which have nanosized dimensions with the help two novel techniques; in-situ and ex-situ technique. On the basis of available literature, we review some of these pioneering studies in which nanocomposities have been evaluated as potential antibacterial activity. Few investigations are reviewed and discussed related to develop for biomedical application especially antibacterial activity of metalpolymer nanocomposities.

In 2011, Mamaghani et al. has reported synthesis of latex based antibacterial acrylate polymer/nanosilver by in-situ technique and its uses as antibacterial agent. The silver nanoparticles were incorporated into poly- 
(methyl methacrylate-butyl acrylate-acrylic acid) using two different approaches. The first approach was based on dispersing the nanosilver particles in acrylic latex and it was synthesized by the emulsion polymerization. The second is the in-situ polymerization of acrylate monomers in the presence of silver nanoparticles by miniemulsion polymerization. Miniemulsion polymerization can yield a better dispersion of nanosilver in the polymeric particles because organic particles can be dispersed directly in the monomer droplets becoming encapsulated upon polymerization.

Morphological investigations were performed using SEM and TEM. FTIR and thermal analyses revealed the silver nanoparticles to be located in the polymeric structure of latex. The nanocomposite latex was compared with the neat latex of the same monomer structure. A comparison of these results showed an increase in the degradation temperature and glass transition temperature compared to the blank latex. The antibacterial properties were determined using the disc diffusion method against both gram-positive (Staphylococcus aureus) and gram-negative (Escherichia coli). The nanocomposite synthesized via in-situ miniemulsion polymerization showed highly potent antibacterial activity toward both grampositive and gram-negative bacteria comparing the neat latex and the blended nanocomposite, which makes it useful for a wide range of biomedical and general applications [3].

Kamrupi et al. (2011) reported a novel method based on ex-situ dispersion of silver nanoparticles within the monomers and subsequent emulsion polymerization using water-in-sc- $\mathrm{CO}_{2}$ medium has introduced in this study. Silver nanoparticles were synthesized by chemical reduction of silver nitrate using sodium borohydrate as a reducing agent and polydimethylsiloxane (PDMS) as a stabilizer in the water-in-sc- $\mathrm{CO}_{2}$ medium. The stable dispersion of silver nanoparticles was added slowly during the polymerization of styrene in the water-in-sc$\mathrm{CO}_{2}$ maintaining the temperature at $70^{\circ} \mathrm{C}$ and pressure at $20.68 \mathrm{MPa}$, respectively. The silver nanoparticles encapsulated within polymer particles were characterized by UVvisible spectroscopy, XRD, TGA, SEM and TEM. The silver/polystyrene nanocomposite particles exhibited antimicrobial activity against a number of bacteria. The current work represents a simple, reproducible and universal way to prepare a variety of metalpolymer nanocomposite particles [4].

Recently, Balan et al. (2012) reported an improvement of the photochemically assisted synthesis of silver nanoparticles by direct photoreduction of $\mathrm{AgNO}_{3}$ with a laser source emitting in the near infrared range (NIR). For this, polymethine dyes were used as the photoactive agents. Both the effects of central chain structure and activation intensity were investigated. The reduction kinetics was followed up by UV-Vis spectroscopy, and the particles size was evaluated by transmission electron microscopy. The light intensity affects both the average size and size distribution of Ag nanoparticles generated through this process. The particles can also be generated in-situ in a photopolymerizable formulation so that metal/polymer nanocomposites become available through a one-step photoassisted process on the basis of NIR activation. The process described herein is very fast (seconds to a few minutes), and it readily lends itself to automatization for mass production of micro-optical elements implemented directly onto integrated NIR sources. This achievement should open up new vistas in several research 
fields, where the in-situ photochemical synthesis of metal/polymer nanocomposites triggered by NIR might allow technical bottlenecks to be overcome [17]. In 2013, Babu et al. reported that sodium carboxymethyl cellulose/poly (acrylamideco-2-acrylamido-2-methylpropane sulfonic acid) semi-interpenetrating polymer network (semi-IPN) hydrogels were prepared by using free radical polymerization technique. Silver nanoparticles were formed by reduction of silver nitrate in semi-IPN hydrogels with sodium borohydrate at room temperature. Various techniques (UV-visible spectroscopy, thermogravimetrical analysis, X-ray diffractometry, SEM and TEM) were used to characterize the formation of silver nanoparticles in hydrogels. SEM images indicated clearly the formation of group of silver nanoparticles with size range of 10 to $20 \mathrm{~nm}$. The sizes of silver nanoparticles were also supported by transmission electron microscopy results. The semi-IPN silver nanocomposite hydrogels reported here might be a potentially smart material in the range of applications of antibacterial activity [18].

The nanocomposities with biomedical investigations are an endless and continuous development process with recent trends in nanotechnology field. It has recently emerged as the most commercially viable technology of this century because of its wide-ranging applications for human being. Man-made nanostructured materials such as fullerenes, nanoparticles, nanopowders, nanotubes, nanowires, nanorods, nanofibers, quantum dots, dendrimers, nanoclusters, nanocrystals, and nanocomposites are globally produced in large quantities due to their broad and potential biomedical applications, e.g., in skincare and consumer products, healthcare, biotechnology, pharmaceuticals and drug delivery. In 2007, Singh and Nalwa summarized that nanoparticles may have adverse biological effects on human and animal health [19]. Since a very wide variety of nanomaterials exit, their interactions with biological systems depend upon their properties. The majority of cytotoxicity studies were done in vitro, which may not give precise information in the comparative toxicity studies in vivo. It is not very clear if nanoparticles induce greater toxic effects to human health than that of microparticles and bulk particles. The effect of nanoparticles on health is an important issue. The toxicity of nanomaterials could be reduced or eliminated by chemical approaches such by surface treatment, functionalization, and composite formation. The toxicity of nanomaterials is a newly emerging research topic based on scientific curiosity raised by scientists and the public, which is not fully understood at this time. It would be more appropriate to state that there are not sufficient scientific data available in the literature to have a very clear picture about the toxic effects of nanomaterials on human health. This is a new emerging area of research lacking sufficient and convenient testing methods, therefore more scientific research is required to understand the correlation between nanomaterials and assess their interactions with biological systems and potential risks on human health. In recent years, nanovaccines have the potential to revolutionize and covers broad spectrum of animal diseases. Dendritic-cell targeted antigen delivery systems hold promise for enhancing vaccine efficacy and delivery of therapeutics. Since decades there has been a considerable research interest in the area of developing drug delivery using nanoparticles as carriers for small and large molecules. Kumar et al. (2013) summarized 
update information on potentiality and remarkable importance of newly developed and tremendous approach of the nanoparticles towards formulation of the nanovaccines [20].

\section{CONCLUSION AND FUTURE DIRECTION}

Studies regarding the potential applications of the metal-polymer composites and largescale production of the composites materials are underway. Till date, there are huge literatures on synthesis, properties, and applications of different nanocomposites of polymer-metal [21], [22], [23], [24]. Recent literature survey provides information on their synthesis by in-situ and ex-situ techniques and their biomedical applications. Metal-Polymer nanocomposities (MPN) have wide range of antibacterial activity. Two techniques for synthesis of MPN are recently used i.e. in-situ \& ex-situ. The techniques posses potential advantages for applications as these are simple, time saving and environmentally benign. Ex-situ technique has additional advantage i.e. the solvent could be recycled. For better antibacterial activity of MPN, in-situ technique is very useful to provide preparation of MPN with maximum nanoparticle dispersion. Current scenario emphasize on synthesis and antibacterial nanomedicine by in- \& ex-situ techniques for industrial production.

\section{DISCLOSURE POLICY}

The authors declare that there is no conflict of interests regarding the publication of this article.

\section{REFERENCES}

1. Hula RA, Pochan DJ. Polymer nanocomposites for biomedical applications. MRS Bulletin. 2007; 32:354358.

2. Yacoby I, Benhar I. Antibacterial nanomedicine. Nanomedicine. 2008; 3(3):329-341.
3. Mamaghani M, Pishvaei YM, Kaffashi B. Synthesis of Latex Based Antibacterial Acrylate Polymer/Nanosilver via In Situ Miniemulsion Polymerization. Macromolecular Research. 2011; 19(3): 243-249.

4. Kamrupi IR, Phukon P, Konwer BK, Dolui SK. Synthesis of silver polystyrene nanocomposite particles using water in supercritical carbon dioxide medium and its antimicrobial activity. Journal of Supercritical Fluids. 2011; 55:1089-1094.

5. Klabunde KJ. Nanoscale Materials in Chemistry. Wiley-Interscience, NewYork; 2001.

6. Gubin SP. Metal containing nano-particles within polymeric matrices: preparation, structure, and properties. Colloids \& Surfaces. 2002; 202:155-163.

7. Mallick K, Witcomb MJ, Scurrell MS. In situ synthesis of copper nanoparticles and poly (o-toluidine): A metalPolymer composite material. Eur Polymer J. 2006; 42: 670-675.

8. He X, Shi O, Zhou X, Wan C, Jiang C. In situ composites of nano SiO2-P (VDF-HFP) porous polymer electrolytes for Li-ion batteries. Electrochemica Acta. 2005; 51: 1069-1075.

9. Nesher G, Marom G, Avnir D. Metal-Polymer compositions: Synthesis and characterization of polyaniline and other polymer @ silver composites. Chem Mater. 2008; 20: 4425-4432.

10. Gubin SP. Metalcontaining nano-particles within polymeric matrices: preparation, structure, and properties. Colloids \& Surfaces. 2002; 202: 155-163.

11. Mayer ABR, Mark JE. Colloidal gold nanoparticles protected by water soluble homopolymers and random copolymers. Eur Polym J. 1998; 34(1):103-8.

12. Kumar RV, Mastai Y, Diamant Y, Gedankon A. Sonochemical synthesis of amorphous $\mathrm{Cu}$ and nanocrystalline $\mathrm{Cu}_{2} \mathrm{O}$ embedded in a polyanilline matrix. J Mater Chem. 2001; 11: 1209-1213.

13. Gangopadhyay $\mathrm{R}$, De A. Conducting polymer nanocomposites: A brief overview. Chem Mater. 2000; 12(3): 608-622.

14. Kaushik R, Kumar A, Sharma N K. Application of Scanning Electron Microscopy in Nanostructure of Polymer-Metal Nanonomposites. ISST J Appl Chem. 2011; 2(2): 63-68.

15. Zezin AB, Rogacheva VB, Feldman VI, Afanasiev P, Zezin AA. From triple interpolyelectrolyte-metal complexes to polymer-metal nanocomposites. Adv Colloid Interface Sci. 2010; 158: 84-93. 
16. Mai YW, Yu Z Z. Polymer Nanocomposites. Woodhead Publishing Limited; 2006.

17. Balan L, Schneider R, Turck C, Lougnot D, MorletSavary F. Photogenerating Silver Nanoparticles and Polymer Nanocomposites by Direct Activation in the Near Infrared. J Nanomaterials. 2012: 1-6.

18. Babu AC, Prabhakar MN, Babu AS, Mallikarjuna B, Subha MCS, Rao KC. Development and Characterization of Semi-IPN Silver Nanocomposite Hydrogels for Antibacterial Applications. Internat J of Carbohydrate Chem. 2013: 1-8.

19. Singh S, Nalwa HS. Nanotechnology and health safety--toxicity and risk assessments of nanostructured materials on human health. J Nanosci Nanotechnol. 2007; 7(9):3048-3070.

20. Kumar A, Sharma NK, Kaushik R. Synthetic Nanoparticles for Nanovaccine". Ann Rev Pharmaceut Sci. 2013; 2(1).

21. Tang E, Liu H, Sun L, Zheng E, Cheng G. Fabrication of Zinc oxide/poly(styrene grafted nanocomposite latex and its application dispersion. Eur Polymer J. 2007; 43: 4210-4218.

22. Nesher G, Marom G and Avnir D. Metal-Polymer compositions: Synthesis and characterization of polyaniline and other polymer @ silver composites. Chem Mater. 2008; 20: 4425-4432.

23. Berkel KYV, Hawker CJ. Tailored composite polymer-metal nanoparticles by miniemulsion polymerization and thiol-ene fictionalization. J Polymer Sci Part A: Polymer Chem. 2010; 48: 1594-1606.

24. Luechinger N A, Grass RN, Athanassiou EK, Stark WJ. Bottom-up fabrication of metal/metal nanocomposites from nanoparticles of immiscible metals. Chem Mater. 2010; 22: 155-160. 